\title{
ANALITYCAL SOLUTION OF THE ONE DIMENSIONAL NONLINEAR TRANSIENT HEAT CONDUCTION PROBLEM USING GREEN'S FUNCTIONS
}

\author{
S. S. Ribeiro ${ }^{a}$, \\ G. C. Oliveira ${ }^{a}$, \\ J. R. F. Oliveirab, \\ and G. Guimarães ${ }^{b}$ \\ aState University of Minas Gerais, \\ Ituiutaba, Minas Gerais, Brazil. \\ ${ }^{\text {b}}$ Federal University of Uberlândia \\ School of Mechanical Engineering \\ Santa Monica, Uberlândia, Minas Gerais, \\ Brazil. \\ sidney.silveira@uemg.br \\ Received: Mar 02, 2021 \\ Revised: Apr 01, 2021 \\ Accepted: Apr 02, 2021

\section{NOMENCLATURE} \\ T temperature, ${ }^{\circ} \mathrm{C}$ \\ $\mathrm{k}(\mathrm{T})$ temperature thermal conductivity dependent, \\ $\mathrm{W} /(\mathrm{mK})$ \\ $\mathrm{k}_{0} \quad$ reference thermal conductivity, $\mathrm{W} /(\mathrm{mK})$ \\ L length, $m$ \\ q heat flux, $\mathrm{W} / \mathrm{m}^{2}$ \\ $\mathrm{t}$ time, $\mathrm{s}$ \\ G Green function \\ $c_{p}(T)$ temperature specific heat capacity dependent, \\ $\mathrm{J} / \mathrm{KgK}$ \\ A real constant \\ $\mathrm{x} \quad$ cartesian coordinate, $\mathrm{m}$
}

\begin{abstract}
Analytical solutions showed to be an important and strong tool for understand thermal problems using mathematic tools. In this work we propose an approach about one dimensional analytical solution for a nonlinear transient heat conduction problem, were used mathematical elements such as Kirchhoff transformation, Green's functions and the combination of them. The combination of this two methods showed that was possible to determinate an analytical solution for the nonlinear thermal problem, and showed a good approximation when compared with results from numerical methods.
\end{abstract}

Keywords: nonlinearity, heat conduction, Kirchhoff transformation, Green's functions

\section{Greek symbols}

$\alpha(\mathrm{T})$ temperature thermal diffusivity dependent, $\mathrm{m}^{2} / \mathrm{s}$

$\beta \quad$ eigenvalue

$\tau \quad$ delayed time

$\rho(\mathrm{T})$ temperature density dependent, $\mathrm{kg} / \mathrm{m}^{3}$

\section{INTRODUCTION}

Analytical solutions shows an important tool for develop solutions on engineering problems, once that analytical solution can be used for validate numerical solutions or, approximated solutions, that makes it easy to analyze and understand physical problems (Fernandes, 2009). The complexity of thermal model from the point of view of obtaining analytical solutions, usually in transient multidimensional problems submitted to non-homogeneities such as prescribed flow boundary conditions, heat generation or boundary conditions as temperature varying over time and nonlinearities.

Transient, multi-dimensional analytical solutions with heat generation or heat flux using Green's functions were showed by (Cole et al., 2010). The authors present a theoretical development and examples of multi-dimensional application, change of variables, and the Green's function method with finite and semi-infinite geometries.

(Sun et al., 2008) using the Kirchhoff integral transformation, showed a model to estimate levels of concrete chloride concentration exposed to a chloride environment. The one-dimensional model was used, where the chloride coefficient term was concrete concentration dependent. Thus, a model was linearized using Kirchhoff transformation and, using a linearized model it was possible to determine the analytical solution, and after, using the inverse Kirchhoff transformation it was obtained the solution for non linear problem. But was consider a steady state heat conduction equation, it become easily solve this problem.

(Zhang et al., 2013) presented the development of a solution of a nonlinear problem using Green's functions by the artificial parameter method, this method consists in replace the nonlinear terms such that: thermal conductivity, density and specific heat, 
by an artificial parameter, and in this way linearize the thermal model. Comparisons were made between the analytical solution of the problem considering temperature dependent properties and constants in order to analyze the influence of nonlinearity on the thermal behavior.

A method to estimate temperature thermal properties dependent were proposed by (Cui et al., 2012), this method consist in use the inverse solution. Initially the thermal properties it is not required, the unknown values are treated as optimization variables, and the errors are minimized between experimental and calculated temperatures. The least squares method was used. Were showed examples of the method efficiency and several ways of engineering uses. Thus, this paper propose an approach of mathematical analysis show a method to determine an analytical solution of nonlinear transient heat conduction problem using the Kirchhoff transformation and the Green's function method.

\section{THEORY}

\section{The one dimensional nonlinear X22 thermal model}

Heat conduction problems can be described using a notation according to their boundary condition. This notation was proposed by (Cole et al., 2010). Thus we will use this notation that consists by letters $\mathrm{X}, \mathrm{Y}$ and $\mathrm{Z}$ to describe the axis directions and numbers $1,2,3$ for boundary conditions of first, second and third kind respectively.

So the X22 thermal model describe a one dimensional model with boundary conditions of second kind in $x=0$ and $x=L$ where $0 \leq x \leq L$ (Cole et al., 2010).

The problem is described by nonlinear differential equation:

$$
\mathrm{k}(\mathrm{T}) \frac{\partial^{2} \mathrm{~T}}{\partial \mathrm{x}_{2}}=\mathrm{C}(\mathrm{T}) \frac{\partial \mathrm{T}}{\partial \mathrm{t}}
$$

Note that the terms $k(T)$ and $C(T)$ are temperature dependent, that is, nonlinearites. Where $C(T)=\rho(T) C_{\rho} k(T)$. Eq.(1) are Subjected to the boundary conditions:

$$
-\left.k(T) \frac{d T}{d x}\right|_{x=0}=q
$$

and

$$
-\left.k(T) \frac{d T}{d x}\right|_{x=L}=0
$$

Note that the boundary conditions also have nonlinearities.

Under the initial condition:

$$
T(x, 0)=T(x)
$$

Normally, all the analytical methods to solve a differential partial equation needs to use the superposition theorem, but, in this case it is not possible due the nonlinearities. Thus, it is necessary to determine an auxiliary problem.

\section{The auxiliary thermal model problem}

(Carslaw and Jaeger, 1959) defines Kirchhoff transformation as:

$$
\phi(x, t)=k(T)=\frac{1}{k_{0}} \int_{0}^{T} k(\widehat{T}) d \widehat{T}
$$

where $k_{0}$ is the reference thermal condutivity (Kevin R. Bagnall, 2014).

Through Eq.(5) by integral definition, we have:

$$
\frac{\mathrm{d} \phi}{\mathrm{dT}}=\frac{\mathrm{k}(\mathrm{T})}{\mathrm{k}_{0}} \Rightarrow \mathrm{k}(\mathrm{T})=\mathrm{k}_{0} \frac{\mathrm{d} \phi}{\mathrm{dT}}
$$

Considering Eq. (1) given by:

$$
\frac{\partial}{\partial \mathrm{x}}\left(\mathrm{k}(\mathrm{T}) \frac{\partial \mathrm{T}}{\partial \mathrm{x}}\right)=\mathrm{C}(\mathrm{T}) \frac{\partial \mathrm{T}}{\partial \mathrm{x}}
$$

Applying Eq.(6) in left side of Eq.(7):

$$
\begin{aligned}
\frac{\partial}{\partial \mathrm{x}}\left(\mathrm{k}(\mathrm{T}) \frac{\partial \mathrm{T}}{\partial \mathrm{x}}\right) & =\frac{\partial}{\partial \mathrm{x}}\left(\mathrm{k}_{0} \frac{\mathrm{d} \phi}{\mathrm{dT}} \frac{\partial \mathrm{T}}{\partial \mathrm{x}}\right) \\
& \Rightarrow \frac{\partial^{2} \phi}{\partial \mathrm{x}^{2}}
\end{aligned}
$$

Multiplying the right side of Eq. (7) by $\frac{\partial \phi}{\partial \phi}$ and using Eq.(6) again, we get:

$$
C(T) \frac{\partial T}{\partial t} \frac{d \phi}{d \phi} \Rightarrow \frac{k_{0}}{\alpha(T)} \frac{d \phi}{\partial \mathrm{t}}
$$

So, we have the auxiliary problem:

$$
\frac{\partial^{2} \phi}{\partial x^{2}}=\frac{1}{\alpha(T)} \frac{\partial \phi}{\partial t}
$$

Note that, in terms of $\Phi$ Eq. (10) remains nonlinear, but in a weaker form, doing $\alpha(T)=\alpha_{0}$, this approximation was detailed by (Ribeiro, 2019), so we have:

$$
\frac{\partial^{2} \phi}{\partial x^{2}}=\frac{1}{\alpha_{0}} \frac{\partial \phi}{\partial t}
$$


Then Eq.(11) defines a linear auxiliary model of Eq.(1). For boundary conditions we use Eq.(6) in the same way.

$$
\begin{aligned}
-\left.k(T) \frac{d T}{d x}\right|_{x=0}= & \left.q \Rightarrow k_{0} \frac{d \phi}{d x} \frac{d T}{d T}\right|_{x=0} \\
& =\left.q \Rightarrow k_{0} \frac{d \phi}{d x}\right|_{x=0}=q
\end{aligned}
$$

and

$$
-\left.k_{0} \frac{d \phi}{d x}\right|_{x=L}=0
$$

and the initial condition

$$
\phi(x, 0)=\phi(x)
$$

Thus, we can use the Green's functions method for establish the $\phi(x, t)$ solution.

\section{The inverse Kirchhoff transformation}

Starting from:

$$
\phi=k(T)=\frac{1}{k_{0}} \int_{0}^{T} k(\widehat{T}) d \widehat{T}
$$

Considering $k(T)=k_{0}(1+A T)$, where $\mathrm{A}$ is a constant, we have:

$$
\begin{array}{r}
k(T)=\frac{1}{k_{0}} \int_{0}^{T} k_{0}(1+A \widehat{T}) d \hat{T} \Rightarrow \phi(T) \\
=k(T)=\frac{A T^{2}}{2}+T
\end{array}
$$

It is possible to determine $T(x, t)$ solution in terms of auxiliary $\phi(x, t)$ by inverse of $k(T)$ function.

$$
\begin{aligned}
T(x, t)=k^{-1}(\phi) & \Rightarrow T(x, t) \\
& =\frac{1}{A}(\sqrt{1+2 A \phi(x, t)} \\
& -1)
\end{aligned}
$$

The Eq.(17) is a analytical solution to the nonlinear problem. Observe that $\mathrm{T}$ is dependent of $\mathrm{A}$, it means, the $\mathrm{T}$ solution indirectly depends of $\mathrm{A}$ that give $k(T)$ behavior

\section{X22 NONLINEAR THERMAL PROBLEM}

The solution of auxiliary problem using Green's functions is given by

$$
\phi(x, t)=\phi_{0}+\frac{\alpha_{0}}{k_{0}} \int_{0}^{\tau} G_{x 22}\left(x, t \mid x^{\prime}, \tau\right) q d \tau
$$

Where $G_{\mathrm{x} 22}\left(\mathrm{x}, \mathrm{t} \mid \mathrm{x}^{\prime}, \tau\right)$ it is the $\mathrm{X} 22$ Green's function (Cole et al., 2010), it is given by

$$
\begin{aligned}
& G_{x 22}\left(x, t \mid x^{\prime}, \tau\right) \\
& =\frac{1}{L} \\
& +\frac{2}{L} \sum_{0}^{\infty} e^{-\beta_{m}^{2} \alpha_{o}(t-\tau)} \cos \left(\beta_{m} x\right) \cos \left(\beta_{m} x^{\prime}\right)
\end{aligned}
$$

Thus, replacing Eq.(19) into Eq.(18) and perform the temporal integration we get

$$
\begin{aligned}
& \phi(x, t) \\
& =\phi_{0}+\frac{q \alpha_{0} t}{k_{0} L}+\frac{2 q}{k_{0} L} \sum_{0}^{\infty} \frac{\cos \left(\beta_{m} x\right)}{\beta_{m}^{2}} \\
& -\frac{2 q}{k_{0} L} \sum_{0}^{\infty} \cos \left(\beta_{m} x\right) \frac{e^{-\beta_{m}^{2} \alpha_{o} t}}{\beta_{m}^{2}}
\end{aligned}
$$

Where $\beta_{m}=\frac{m \pi}{L}$ are eigenvalues

Thus

$$
\begin{aligned}
& T(x, t)=k^{-1}(\phi) \Rightarrow T(x, t) \\
& =\frac{-1+\sqrt{2 A \phi(x, t)+1}}{k_{0}}
\end{aligned}
$$

Once again note that $\mathrm{T}$ solution depends of constant A, reference thermal conductivity and the auxiliary solution $\phi$.

In the next section the solution Eq.(21) will be computed and compared with the FEA method by software Comsol.

\section{RESULTS AND DISCUSSION}

By analytical nonlinear solution through open source software Scilab, the Eq.(21) were computed and compared with the linear solution version and numerical solution that is given by finite element method (FEA) by software Comsol Multiphysics.

Then, using a one dimensional geometry with length $\mathrm{L}=0,01[\mathrm{~m}]$, the temperatures were computed at the $\mathrm{x}=0[\mathrm{~m}]$ point. The thermal properties were $\mathrm{k}_{0}=14,9\left[\mathrm{Wm}^{-1} \mathrm{~K}^{-1}\right], \quad \alpha_{0}=3,95 \times 10^{-6}\left[\mathrm{~ms}^{-2}\right]$ and $\mathrm{k}(\mathrm{T})=\mathrm{k}_{0}(1+\mathrm{AT})$, the constant heat flux applied was $\mathrm{q}=10^{5}[\mathrm{~W}]$ at $\mathrm{x}=0[\mathrm{~m}]$ position. The initial condition, $\mathrm{T}(\mathrm{x}, 0)=30\left[{ }^{\circ} \mathrm{C}\right]$ and $\mathrm{A}=1 \times$ $10^{-3}$. These values were chosen hypothetically.

The Fig.(1) shows the behavior of temperature thermal conductivity, we can see that the thermal conductivity increase with the temperatures, this is due to the choice of constant $\mathrm{A}$, it means $\mathrm{A}$ is greater than zero. For different choices of A we have different behaviors for the thermal conductivity. 


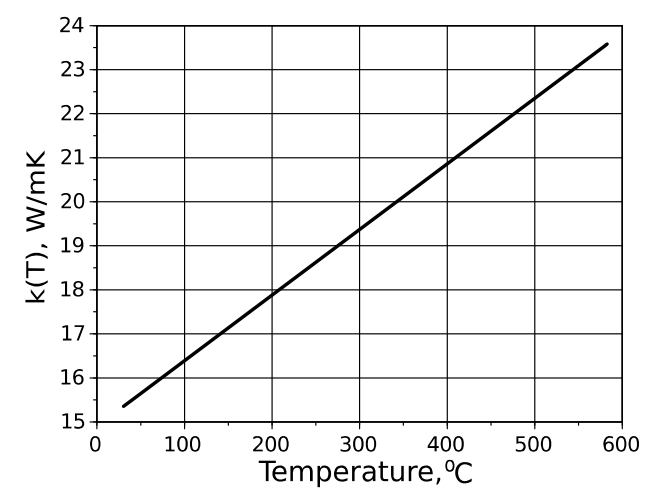

Figure 1. Behavior of temperature thermal conductivity dependent.

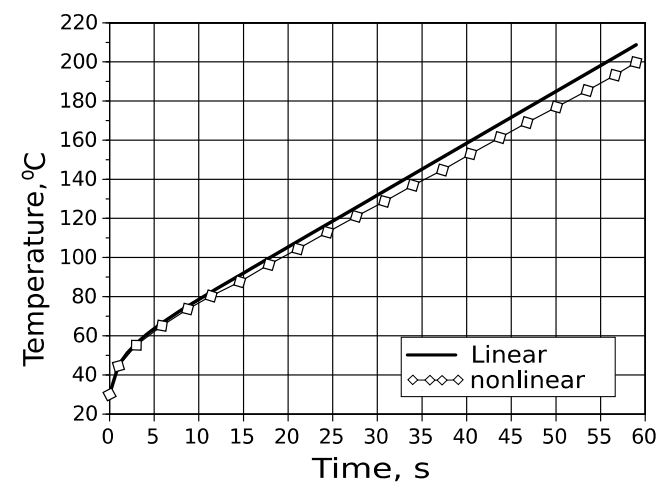

Figure 2. Comparison between linear and nonlinear version of thermal problem.

The generated temperatures by nonlinear and linear model was presented by Fig(2). Note that the temperatures increase from the initial condition during $60 s$, this simulate time was chosen because it is enough for observe the thermal phenomena. It can be observe too, that nonlinear temperatures are less than linear temperatures, this fact is occurs due the $k(T)$ effect. It is observed that the linear version problem is given by $\phi(x, t)$.

Another comparison was made using temperatures generated by Consol Multiphysics software. Consol uses the FEA method to calculate the temperatures. This temperatures are shown by Fig.(3), we can see a great agreement between two thermal profiles, a little bit difference can be observed from $40 s$ it is occour due Comsol uses the function $k(T)$ to calculate the thermal diffusivity, it means that thermal diffusivity still remains nonlinear in Consol environment, what does not occur in the analytical solution. But, we can be observe that the thermal diffusivity does not present a great impact to numerical temperatures.

To show numerically the differences between analytical and numerical solutions we can see the Fig.(4). We can observe that all differences were less than $2,7 \%$. This fact shows that two solutions are very close. Notice that, the numerical solution, in this case, was not used to validate the analytical solution, was used only for comparison criteria.

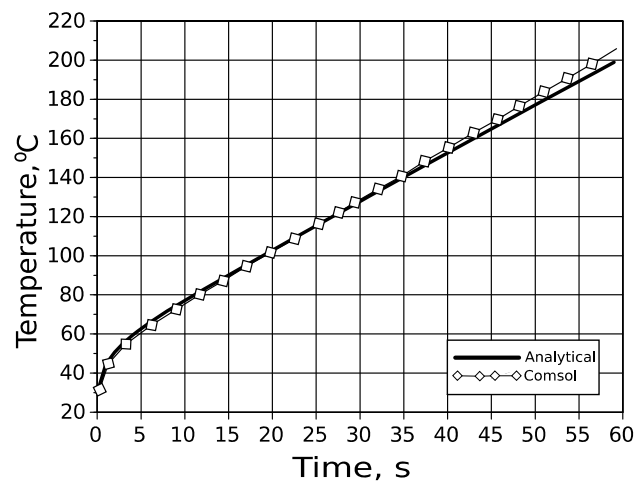

Figure 3. Comparison between analytical and numerical solution.

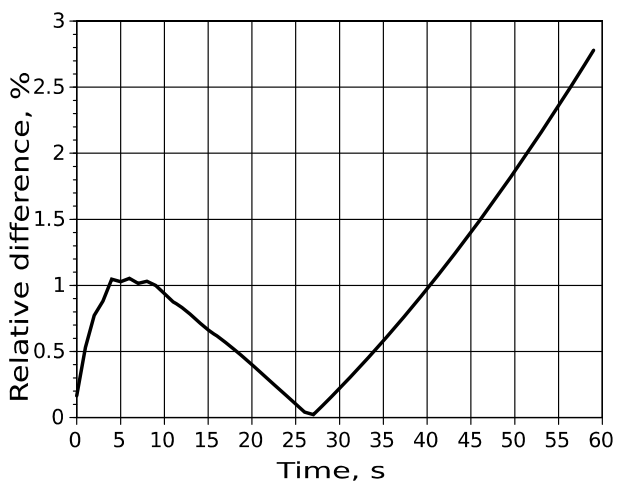

Figure 4. Relative difference calculated when compared analytical and numerical solutions.

\section{CONCLUSIONS}

This work proposed a way to solve the transient heat conduction using Green's functions. Was showed the mathematical analysis and Kirchhoff variables changes to obtain a linear model version with the boundary conditions, and so it was made possible to apply the Green's function method due the possibility of use the superposition theorem.

After returning to the original problem using the inverse Kirchhoff transformation it was possible to show a graphical problem solution and made comparisons with a numerical solution by Comsol. The thermal profile presented a great approximation when compared to the numerical solution, where all differences less than $2.7 \%$.

It is observed too that analytical solutions are very easy to be implemented in softwares like Matlab, Scilab, Octave, Phyton, etc. and presented a short execution time when compared with numerical methods. Other fact is, analytical solutions are exact, it means, does not depend on rounding or truncation, 
which makes it a strong tool, and, a comprehension of one dimensional analytical solution can be a facilitator to understand and solve two and three dimensional nonlinear heat problems.

\section{ACKNOWLEDGEMENTS}

The authors would like thank to the Brazilian funding agencies CAPES, FAPEMING and CNPq.

\section{REFERENCES}

Carslaw, H.S. and Jaeger, J.C., 1959, Conduction of Heat in Solids. Clarendon Press, Oxford, 2nd edition.

Cole, K.D., Beck, J.V., Haji-Sheikh, A. and Litkouhi, B., 2010, Heat Conduction Using Green's Functions. Series in computational and physical processes in mechanics and thermal sciences, Taylor \& Francis Group. ISBN 9781439813546.

Cui, M., Gao, X. and Zhang, J., 2012, A new approach for the estimation of temperature-dependent thermal properties by solving transient inverse heat conduction problems, International Journal of Thermal Sciences, Vol. 58, p. 113-119.

Fernandes, A.P., 2009, Funções de Green: Soluções analíticas aplicadas a problemas inversos em condução de calor, Master's thesis, Universidade Federal de Uberlandia, Uberlandia. URL http://www.bdtd.ufu.br.

Kevin R. Bagnall, Yuri S. Muzychka, E.N.W., 2014. Application of the Kirchhoff transform to thermal spreading problems with convection boundary conditions, IEEE Transactions on Components, Packaging and Manufacturing Technology, Vol. 4, No. 3, p. 408-420.

Ribeiro, S.S., 2019, Uso da transformada de Kirchhoff e funções de Green para solução analítica de problemas inversos não lineares em condução de calor, Doctorate's thesis, Universidade Federal de Uberlandia, Uberlandia. URL https://repositorio.ufu.br/bitstream/123456789/29059/ 1/UsoTransformadaKirchhoff.pdf

Sun, Y.M., Chang, T.P. and Liang, M.T., 2008, Kirchhoff transformation analysis for determining time/deph dependent chloride diffusion coefficient in concrete, Springer Science + Business Media.

Zhang, H., Kan, W. and Hu, X., 2013, Green's function approach to the nonlinear transient heat transfer analysis of functionally graded materials, International Journal of Thermal Sciences, Vol. 71, p. 292-301. 